\title{
Recurrent Painful Ophthalmoplegic Neuropathy with Residual Mydriasis in an Adult: Should it Be Classified as Ophthalmoplegic Migraine?
}

\author{
Yuya Kobayashi, Yasufumi Kondo, Kana Uchibori and Jun Tsuyuzaki
}

\begin{abstract}
:
Recurrent painful ophthalmoplegic neuropathy (RPON) is a rare condition that manifests as headache and ophthalmoplegia. It typically occurs in children. Although migraine or neuropathy have been suggested as etiologies, the precise etiology remains unclear. In the International Classification of Headache Disorders 3rd edition-beta version (ICHD3 $\beta$ ) (code 13.9), RPON was categorized into painful cranial neuropathies and other facial pains. We encountered a 48-year-old woman who had diplopia and right ptosis. The administration of prednisolone led to the immediate improvement of her oculomotor palsy, but residual mydriasis remained. Based on this case, the pathophysiology of RPON may involve temporary nerve inflammation with migraine. Repeated and severe migraine attacks may cause irreversible nerve damage. Thus, medication for migraine prophylaxis might be needed to prevent RPON.
\end{abstract}

Key words: recurrent painful ophthalmoplegic neuropathy, ophthalmoplegic migraine, migraine, oculomotor palsy, mydriasis, headache

(Intern Med 56: 2769-2772, 2017)

(DOI: 10.2169/internalmedicine.8842-17)

\section{Introduction}

Abnormal eye movement is often an early symptom of a critical neurological disease. Recurrent painful ophthalmoplegic neuropathy (RPON) is a rare condition involving headache and ophthalmoplegia, which typically occurs in children $(1,2)$. Its annual incidence is estimated to be 0.7 per million (3) and it is usually not easy to diagnose in adult cases. The International Classification of Headache Disorders 3rd edition-beta version (ICHD3 $\beta$ ) defined the following diagnostic criteria for RPON: 1) at least two attacks; 2) unilateral headache accompanied by ipsilateral paresis of one, two, or three ocular motor nerves; 3) the exclusion of orbital, parasellar, or posterior fossa lesions by an appropriate investigation; and 4) the absence of another diagnosis that could better account for the patient's condition (4).

RPON had been previously known as ophthalmoplegic migraine $(\mathrm{OM})$, but the disease concept has changed due to the fact that it closely resembles recurrent painful neuropathy and because some cases occur without migraine symptoms (5). The etiology remains unclear and it has been difficult to collect case series and pathologically proven cases.

We encountered an adult patient with prolonged RPON with migraine who improved rapidly with steroids, but who showed residual mydriasis. We aimed to report the etiological association between RPON and migraine in such cases. This case raises a criticism regarding the classification of RPON in view of the lack of association with migraine.

\section{Case Report}

The patient was 48-year-old Japanese woman. She reported experiencing isolated migraine attacks every 1-2 months with occasional vomiting in her 20's. Her family history and other medical history were unremarkable. She recalled having two episodes of diplopia and right ptosis with severe migraine at 32 years and 38 years of age, respectively. In both instances, the diplopia and right ptosis 


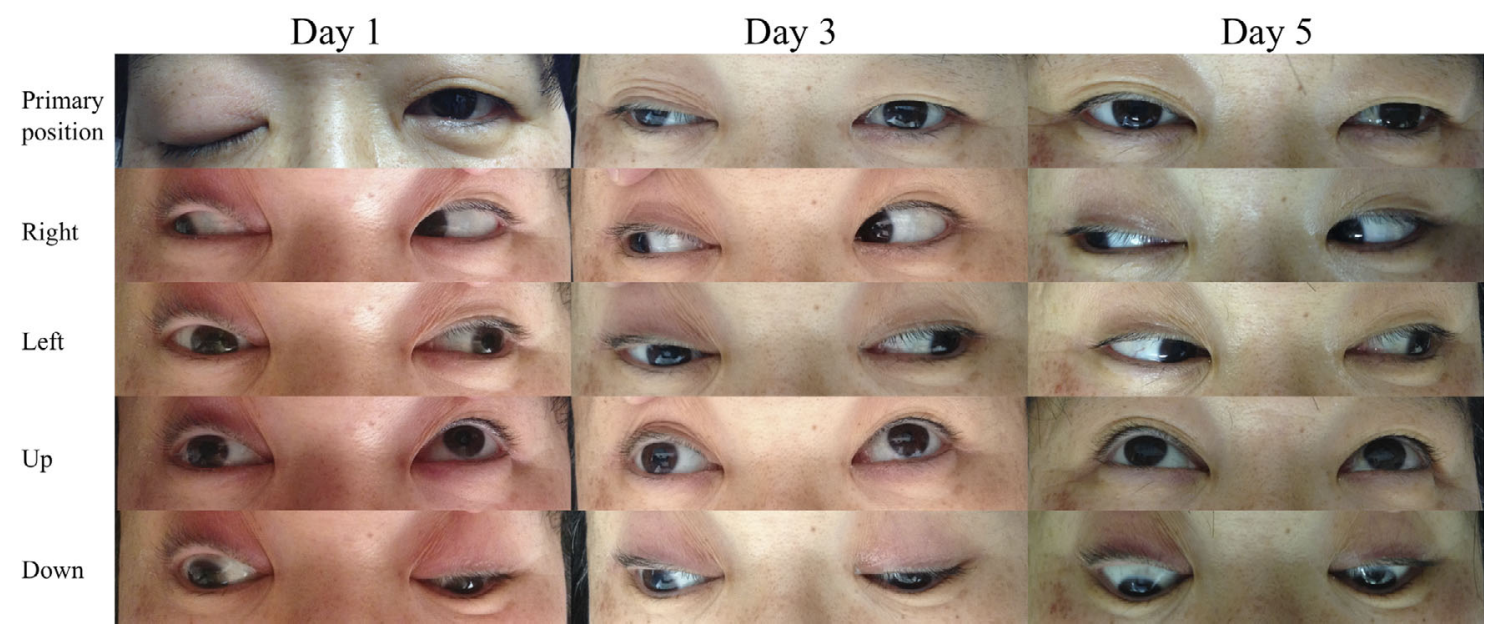

Figure 1. An adult woman with right-sided ptosis and third cranial nerve palsy. She was treated with prednisolone, and her condition gradually improved. When not in the primary position, her eyes were opened by external force.

developed a few days after the migraine attack and the symptoms resolved spontaneously after a few weeks.

At this time, she was admitted to our hospital with headache and vomiting, followed by diplopia, right-sided ptosis and photophobia two days later. She suffered from extreme fatigue and stress, similar to the previous two episodes. Her migraine attack was the worst one that she had ever experienced. Her headache occurred on the right side and was accompanied by right orbital pain with visual aura, pulsating pain, photophobia and phonophobia, all of the symptoms matched the diagnostic criteria of migraine. Her condition lasted for three days and was worsened by routine physical activities. Her vital signs and the results of a physical examination were normal. She had no symptoms of infection. A neurological examination revealed right-sided ptosis, divergent squint, and outward and downward displacement of the right eye (Fig. 1). The right eye had negative direct and indirect pupillary reflexes; her pupil size was $5 \mathrm{~mm} / 3 \mathrm{~mm}$ (right/left).

Laboratory analyses, including a routine hemogram, and the measurement of her blood sugar level, thyroid hormone levels and erythrocyte sedimentation rate were nonspecific. Tests for myeloperoxidase antineutrophil cytoplasmic antibody (ANCA) and cytoplasmic ANCA were negative, and her angiotensin-converting enzyme (ACE) and lysozyme levels were $11.5 \mathrm{U} / \mathrm{L}$ and $4.1 \mu \mathrm{g} / \mathrm{mL}$, respectively. A cerebrospinal test revealed 2 mononuclear cells $/ \mathrm{mm}^{3}$, a protein level of $21 \mathrm{mg} / \mathrm{dL}$, and a glucose level of $71 \mathrm{mg} / \mathrm{dL}$. T1-weighted magnetic resonance imaging (MRI) with gadolinium enhanced the oculomotor nerve at its exit from the brainstem, no other abnormal other sequences were observed (Fig. 2). The magnetic resonance angiography findings were normal. No enhancement of the cavernous sinus, adjacent dura, or abnormal blood vessels was observed. We excluded other causes of focal cranial neuropathy including vascular, inflammatory, tumor-related, metabolic, autoimmune, and infectious disorders. Her symptoms met the diagnostic criteria of RPON in ICHD-3 3 (4), and treatment was initiated with prednisolone (30 mg/day), which was subsequently tapered. Her eye movement improved on day 5 (Fig. 1); however, the dilation of the right pupil and photophobia were still present at a 3-year follow-up examination (Fig. 3). The eye movement was normal, but no miosis in the right eye was observed in near reflex in convergence. Follow-up MRI with gadolinium on day 8 and 4 months later showed the disappearance of the contrast effect (Fig. 2).

\section{Discussion}

Our patient experienced three episodes of oculomotor palsy. RPON is reported to be monophasic in $77.4 \%$ of patients; the remaining patients experience two or more attacks (6). The patient suffered from persistent mydriasis after her third attack. In $30 \%$ of the cases, recurrent episodes of RPON (or OM) may be severe enough to cause permanent neurologic sequelae (1). The development of mydriasis has been reported to occur in $28.6-93.8 \%$ of patients $(2,6,7)$. The pupils are regulated by parasympathetic fibers and are located on the outer layer of the oculomotor nerve. In patients of oculomotor palsy due to cerebral aneurysm, mydriasis may occur depending on the shape, position, and size of the aneurysm (8). In other words, the development of mydriasis due to RPON may depend on the involved site. Thus, in our patient, repeated inflammatory attacks may have damaged the outer layer of the oculomotor parasympathetic nerve fiber.

In general, RPON is not associated with any characteristic blood or cerebrospinal fluid abnormalities. In 75-100\% of patients, T1-weighted MRI with gadolinium during an acute attack demonstrates cranial nerve enhancement $(1,5,9)$. In our case, the oculomotor nerve was enhanced on T1weighted MRI in the earlier stages of the disease, and the enhancement disappeared without the symptomatic recovery of the pupil. The MRI finding was transient and was indica- 

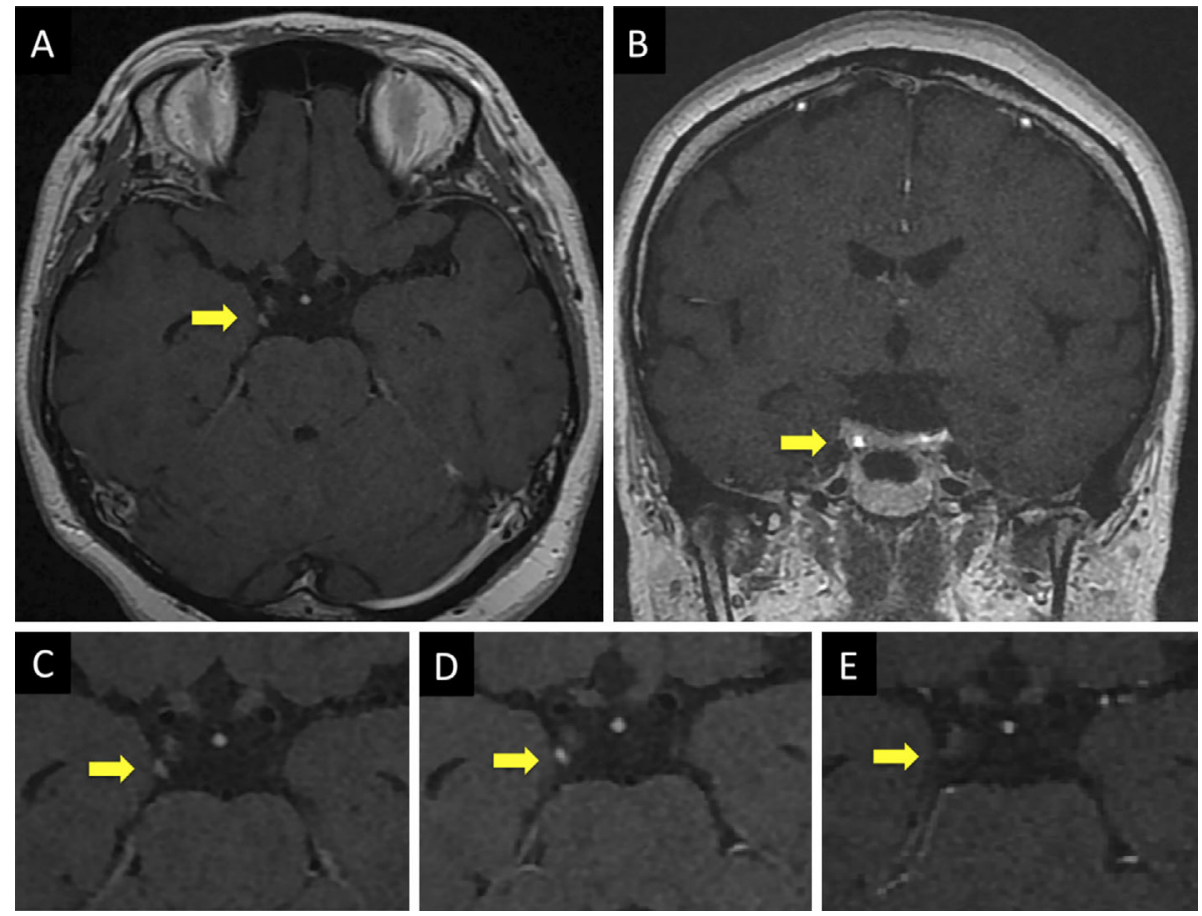

Figure 2. The serial findings on MRI with gadolinium in an adult woman with recurrent painful ophthalmoplegie neuropathy. (A, B, C) On admission, the oculomotor nerve (arrows) showed enhancement. MRI with gadolinium on day 8 (D) and at 4 months (E). The disappearance of the contrast effect was observed at 4 months (E).
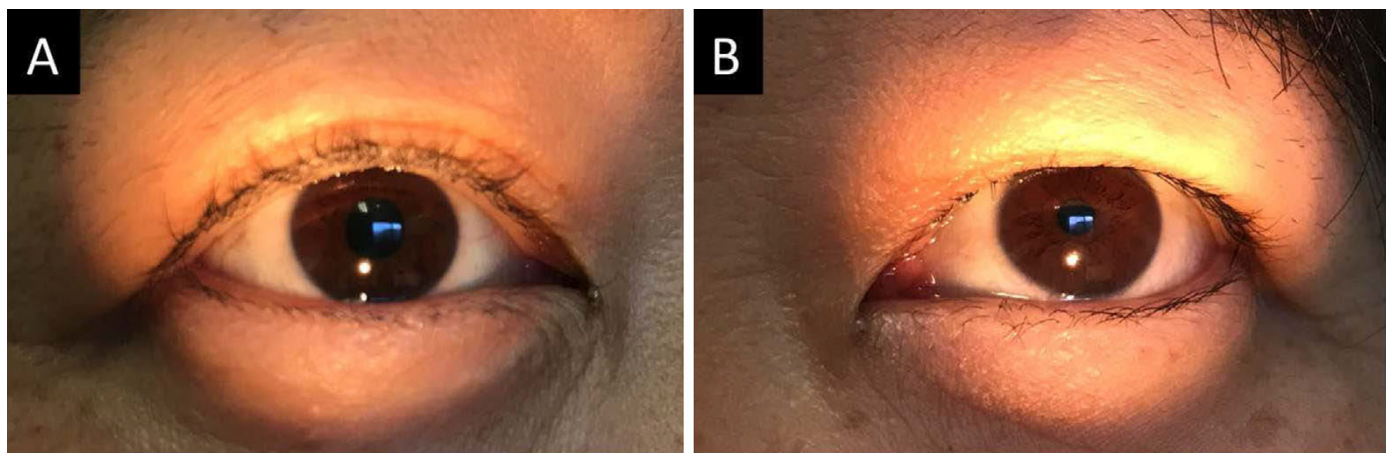

Figure 3. Three years after the resolution of recurrent painful ophthalmoplegie neuropathy. (A) The right pupil remains dilated, (B) whereas the left pupil is normal.

tive of a temporary inflammatory change. Thus, it is better to perform an MRI in the early stage. This MRI finding is not necessary to fulfill the diagnostic criteria for RPON, especially in children. The thickening of the oculomotor nerve at the exit from the midbrain was reported in a small number of autopsied cases (10). Such changes may be caused by inflammation, which may also be captured by contrasted MRI. Although no biopsy was conducted in this case, we believed that irreversible oculomotor parasympathetic nerve damage occurred.

The use of prednisolone in the treatment of RPON has been reported $(1,2,5,6,9)$; however, its effects regarding the long-term prevention of RPON recurrence are uncertain. Similarly, the efficacy of drugs for migraine such as acetaminophen, nonsteroidal anti-inflammatory drugs (NSAIDs), ergotamine and triptans is unknown. Cyproheptadine hydro- chloride has been reported to prevent recurrent attacks of ophthalmoplegic migraine (11) by targeting the vascular edema around the oculomotor nerve.

Our patient only developed mydriasis after an uncontrolled and severe migraine, which was induced by extreme stress and fatigue. This observation was consistent with the results of one report, which showed that $95.2 \%$ of patients developed ophthalmoplegia during a severe migraine attack (6). At present, the patient's symptoms are wellcontrolled by NSAIDs alone. Further, severe attacks of migraine should be prevented by avoiding known triggers and exacerbating factors.

Although OM was first described in 1854 (2), the cause still remains unclear. Theories involving demyelination (12), inflammation, and vasospasm caused by neuropeptide release (9) have been discussed, but these cannot explain the 
repeated occurrences of single-nerve palsy with headache in RPON. Because the symptoms were temporary, there was no chance for pathological examination. In this case, the oculomotor nerve palsy improved more quickly than the other disorders, suggesting that it was initially a transient symptom that occurred with migraine. We believed that the fragile part of the nerve was impaired by inflammatory peptides due to migraine. Various mediators, such as nitric oxide, histamine, serotonin, and calcitonin gene-related peptide are known to be secreted during migraine attacks (13); in severe attacks, these mediators are secreted more excessively than usual and can damage the fragile portion of the same nerve. Moreover, the reason why OM is common in children could be that their cranial nerves are partially undeveloped and fragile.

This syndrome is characterized by recurrent painful neuropathy and is sometimes not migrainous; thus, RPON has been excluded from the disease concept of migraine. For this reason, the old and inappropriate term "OM" is now rejected by some authors (4), but accepted by others (14). In this case, oculomotor nerve palsy developed with migraine and improved faster than any of the other neuropathies, suggesting a diagnosis of OM, not RPON.

Most of the available reports in the literature have not described a clear and single etiology of RPON. Thus, future studies and the accumulation of autopsy cases will be needed to determine the validity of RPON as a clinical entity.

The authors state that they have no Conflict of Interest (COI).

\section{References}

1. McMillan HJ, Keene DL, Jacob P, Humphreys P. Ophthalmoplegic migraine: inflammatory neuropathy with secondary migraine? Can J Neurol Sci 34: 349-455, 2007.

2. Carlow TJ. Oculomotor ophthalmoplegic migraine: is it really migraine? J Neuroophthalmol 22: 215-221, 2002.

3. Hansen SL, Borelli-Møller L, Strange P, Nielsen BM, Olesen J.
Ophthalmoplegic migraine: diagnostic criteria, incidence of hospitalization and possible etiology. Acta Neurol Scand 81: 54-60, 1990.

4. Headache Classification Committee of the International Headache Society. The international classification of headache disorders. Cephalalgia 33: 629-808, 2013.

5. Gelfand AA, Gelfand JM, Prabakhar P, Goadsby PJ. Ophthalmoplegic "migraine" or recurrent ophthalmoplegic cranial neuropathy: new cases and a systematic review. J Child Neurol 27: 759-766, 2012.

6. Lal V, Sahota P, Singh P, Gupta A, Prabhakar S. Ophthalmoplegia with migraine in adults: is it ophthalmoplegic migraine? Headache 49: 838-850, 2009.

7. Wong V, Wong WC. Enhancement of oculomotor nerve: a diagnostic criterion for ophthalmoplegic migraine? Pediatr Neurol 17: 70-73, 1997.

8. Motoyama Y, Nonaka J, Hironaka Y, Park YS, Nakase H. Pupilsparing oculomotor nerve palsy caused by upward compression of a large posterior communicating artery aneurysm. Case report. Neurol Med Chir (Tokyo) 52: 202-205, 2012.

9. Mark AS, Casselman J, Brown D, et al. Ophthalmoplegic migraine: reversible enhancement and thickening of the cisternal segment of the oculomotor nerve on contrast-enhanced MR images. AJNR Am J Neuroradiol 19: 1887-1891, 1998.

10. Lee AG. Oculomotor ophthalmoplegic migraine: what really causes it? J Neuroophthalmol 23: 240, 2003.

11. Sugiyama N, Hamano S, Tanaka M, Mochizuki M, Nara T. MRI findings and effectiveness of cyproheptadine in two patients with ophthalmoplegic migraine. No To Hattatsu 34: 533-537, 2002 (in Japanese, Abstract in English).

12. Lance JW, Zagami AS. Ophthalmoplegic migraine: a recurrent demyelinating neuropathy? Cephalalgia 21: 84-89, 2001.

13. Gupta S, Nahas SJ, Peterlin BL. Chemical mediators of migraine: preclinical and clinical observations. Headache 51: 1029-1045, 2011.

14. Wang Y, Wang XH, Tian MM, et al. Ophthalmoplegia starting with a headache circumscribed in a line-shaped area: a subtype of ophthalmoplegic migraine? J Headache Pain 15: 19, 2014.

The Internal Medicine is an Open Access article distributed under the Creative Commons Attribution-NonCommercial-NoDerivatives 4.0 International License. To view the details of this license, please visit (https://creativecommons.org/licenses/ by-nc-nd/4.0/).

(C) 2017 The Japanese Society of Internal Medicine Intern Med 56: 2769-2772, 2017 